\title{
Costo anual per cápita en primer nivel de atención por género
}

\author{
Cost per capita in outpatients by gender
}

Enrique Villarreal-Ríos ${ }^{1}$

Maribel Campos Esparza ${ }^{1}$

Liliana Galicia Rodríguez ${ }^{1}$

Lidia Martínez González ${ }^{1}$

Emma Rosa Vargas Daza ${ }^{1}$

Guadalupe Torres Labra ${ }^{1}$

Adolfo Patiño Vega ${ }^{1}$

María Teresa Rivera Martínez ${ }^{1}$

Raúl Aparicio Rojas ${ }^{1}$

Martín Juárez Durán ${ }^{1}$

${ }^{1}$ Unidad de Investigación

Epidemiológica y en

Servicios de Salud

Querétaro, Instituto

Mexicano del Seguro Social.

Zaragoza y 5 de Febrero,

Colonia Centro, Querétaro,

Querétaro de Arteaga.

México. 76000.

felibree@infosel.net.mx
Abstract The objective of this study is to identify the annual cost per capita by gender in first level of attention. It is a cost study in Family Physician Units in Mexico. The information corresponded to the year of 2004 and the study divided in the use profile and cost attention. Use profile of services: it was studied 1,585 clinical registries of patients, use profile defined by average and attention reasons by department, gender and age group. Cost attention: considered in American dollars it included fixed unit cost (departmentalization adjusted by productivity), variable unit cost (micro cost technical), department unite cost by type attention, and department unit cost by age and gender. The life expectancy was of 73 years for men and 78 for women. Three scenes were identified. The annual cost per capita is superior among woman [US\$73.24 (IC 95\% \$11.38 - \$197.49)] than in man [\$53.11 (IC 95\% 2.51 - 207.71)]. The conclusion found that in the first level of attention the cost per capita is greater in woman than in man.

Key words Cost, Attention, Profile, Gender
Resumen El objetivo de este artículo es estimar el costo per cápita anual por género en primer nivel de atención. Métodos: estudio de costo realizado en Unidades de Medicina Familiar en México. La información correspondió al año 2004 y el estudio se dividió en perfil de uso y costo de la atención. Perfil de uso de lo servicios: se estudiaron los registros clínicos de 1,585 pacientes adscritos a unidades de medicina familiar. El perfil de uso se definió mediante el promedio y los motivos de atención por departamento, género y grupo de edad. Costo de la atención: estimado en dólares estadounidenses incluyó costo unitario fijo (departamentalización ajustada por productividad), el costo unitario variable (técnica de microcosteo), costo unitario por departamento y motivo de atención, y costo unitario por departamento, quinquenio y género. La esperanza de vida fue de 73 años para los hombres y 78 para las mujeres. Se identificaron tres escenarios. Resultados: el costo anual per capita es superior en la mujer US \$73.24 (IC 95\% \$11.38 - \$197.49) que en el hombre \$ 53.11 (IC 95\% 2.51 - 207.71). Conclusión: en primer nivel de atención el costo per cápita en las mujeres es mayor que en los hombres, información valiosa en el proceso de planeación de los servicios de salud.

Palabras clave Costo, Atención, Perfil, Género 


\section{Introducción}

La importancia de conocer los costos radica en la relación que existe con la eficiencia y eficacia y de la mano con índices como costo efectividad y costo beneficio que favorecen una mejor asignación y utilización de los escasos recursos existentes. Esta concepción aplicada a los sistemas de salud públicos rebasa ampliamente el concepto de que conocer los costos tiene por finalidad servir de base para establecer el precio de las prestaciones de salud y se extiende a aspectos como planeación, toma de decisiones y financiamiento ${ }^{1-3}$.

Los métodos para costear son diversos e incluyen costeo directo, predeterminado, por absorción, real normalizado, real integrado y $\mathrm{ABC}$ entre otros. El resultado será distinto con cada uno de ellos pero será el costo de la empresa ${ }^{4,5}$. En el campo de la salud se reporta una gran variación de los costos, por ejemplo por patologías específicas se señala el costo de neoplasias $\$ 35,372$, hipertensión $\$ 5,862$, diabetes $\$ 2,548$ y aun al interior de ellas la variación está presente ${ }^{6-9}$. Cuando el análisis se hace entre grupos etarios ${ }^{10} \mathrm{o}$ al interior de uno de ellos por ejemplo adultos mayores, el costo por quinquenio se modifica y si el análisis se extiende al género la variación persiste ${ }^{6}$.

Otro indicador es el gasto per cápita, en él también existe una amplia variación, cuando se analiza por países se encuentran valores entre (US) $\$ 11$ en Bangladesh, y (US) \$5,274 en Estados Unidos de América ${ }^{11}$. Para el caso de México el gasto per cápita por subsistema de salud (Institución) adopta este mismo comportamiento, la variación identificada se sitúa en el rango de \$1,651 a $\$ 2,606$ (pesos mexicanos) ${ }^{12}$. Al interior de cada subsistema, lo gastado en atención hospitalaria difiere de lo gastado en la atención ambulatoria ${ }^{8}$, y aun en ésta el gasto se diferencia por tipo de servicio, correspondiendo el mayor porcentaje a los servicios curativos ${ }^{13}$. En este contexto el objetivo del trabajo fue estimar el costo per cápita anual por género en primer nivel de atención.

\section{Métodos}

Se trata de un estudio de costo realizado en siete unidades de medicina familiar del área metropolitana de la ciudad de Querétaro, pertenecientes a la Institución de Seguridad Social más grande de México. La información correspondió al año 2004 y el estudio se dividió en perfil de uso y costo de la atención.

\section{Perfil de uso}

El perfil de uso se identificó por cada departamento de la unidad de medicina familiar y se estimó para un año.

- Se estudiaron los registros clínicos de 1,585 pacientes adscritos a unidades de medicina familiar.

- Fueron distribuidos proporcionalmente en cada clínica y al interior de éstas equitativamente entre el total de consultorios.

- La selección al interior del consultorio se realizó aleatoriamente empleando como marco muestral el listado de pacientes existentes.

- El perfil de uso se definió como el promedio anual de uso de cada uno de los departamentos de la unidad de medicina familiar (consulta de Medicina Familiar, Enfermera Materno Infantil, Estomatología, Trabajo Social, Medicina Preventiva, Laboratorio, Radiología, Urgencias, Nutrición y Planificación Familiar) y el motivo de la atención (principales motivos de cada uno de los departamentos estudiados).

- El promedio anual de uso se estimó por género y quinquenio de edad $(0$ a 4,5 a 9,10 a 14 , 15 a 19,20 a 24,25 a 29,30 a 34,35 a 39,40 a 44 45 a 49,50 a 54,55 a 59,60 a 64,65 a 69,70 a 74 y 75 a 78). La esperanza de vida para el hombre se estableció en 73 años y en la mujer 78 años ${ }^{14}$.

- El promedio por departamento se estimó a partir de los promedios de cada uno de los quinquenios.

- El análisis se realizó en dos perspectivas:

. Población total: se incluyeron todos los expedientes independientemente de que hubieran hecho uso de algún departamento en el periodo de estudio.

. Población usuária: se incluyeron exclusivamente los expedientes con reporte de uso de al menos una atención en el departamento en el periodo de estudio.

\section{Costo de la atención}

El costo de la atención se estimó para un año en dólares estadounidenses utilizando la metodología que a continuación se presenta.

\section{Costo unitario fijo por tipo de departamento}

Para el costo unitario fijo se empleó la departamentalización ajustada por productividad anual (total de atenciones otorgadas en cada uno de los departamentos finales). 
- Se identificaron departamentos generales y finales. Se consideraron departamentos generales aquellos sin relación directa con el paciente (Dirección, Administración, Conservación, Archivo). Los departamentos finales se definieron como aquellos con relación directa con el paciente (consulta de Medicina Familiar, Enfermera Materno Infantil, Estomatología, Trabajo Social, Medicina Preventiva, Laboratorio, Radiología, Urgencias, Nutrición y Planificación Familiar).

- Al interior de los departamentos generales y finales se identificaron los insumos requeridos para producir el servicio y fueron agrupados en cuatro: recursos materiales (mobiliario, equipo e instrumental), físicos (infraestructura), humanos (gasto en personal) y de consumo (material de oficina, telecomunicaciones, combustible, energía eléctrica y agua).

- Por cada uno de los insumos identificados se estimó el gasto anual por departamento.

- El gasto en los departamentos finales se calculó con lo gastado directamente más la parte proporcional que le correspondió de cada departamento general. Para asignar los recursos de los departamentos generales a cada uno de los finales se construyeron ponderadores en función del peso específico de cada departamento final para cada tipo de insumo.

- Para los insumos materiales y físicos (mobiliario, equipo, instrumental e infraestructura) se estimó la vida media útil, con la finalidad de poder asignar correctamente a los servicios producidos la parte proporcional de lo gastado en la inversión (total del gasto en inversión/vida media útil) y conocer el gasto anual. Se definió como vida media útil de 20 años para la infraestructura, 10 para mobiliario, cinco para equipo y uno para instrumental.

- La estimación del gasto en personal contempló el salario anual integrado (sueldo base y prestaciones) incluidas las deducciones. Para asignar la parte correspondiente de los departamentos generales a los finales se identificó la suma total de los gastos generados en pago de personal en los departamentos finales y se consideró el 100\%, se identificó qué porcentaje de este gasto correspondía a cada uno de los departamentos finales (por ejemplo 20\% laboratorio), el valor identificado se empleó como ponderador y se aplicó al gasto total anual generado en los departamentos generales (por ejemplo \$100,000), el resultado de la multiplicación $(100,000 * .20=20,000)$ fue la parte proporcional de lo gastado en departamentos generales que le correspondió al departamento de laboratorio y se sumó a lo gastado directamente en personal en este departamento.

- Para asignar a los departamentos finales el gasto en insumos de consumo los ponderadores se construyeron de acuerdo al siguiente criterio: material de oficina (cantidad servicios generados al año en cada departamento final), telecomunicaciones (número de extensiones telefónicas), combustible (metros cuadrados de construcción), energía eléctrica (número de lámparas) y agua (metros cuadrados de construcción).

- Por cada departamento final se sumaron los costos anuales de cada uno de los insumos identificados para establecer el costo total fijo anual del departamento.

- Se identificó la productividad (total de acciones realizadas en un año) por cada uno de los departamentos finales y se dividió entre el costo total fijo anual, para conocer el costo unitario fijo por departamento final.

\section{Costo unitario variable por intervención}

La estimación del costo unitario variable se realizó empleando la técnica de microcosteo.

- Para cada quinquenio de edad se identificaron los principales motivos de atención por departamento.

- El tipo y la cantidad de insumos (medicamento, material de curación y reactivos de laboratorio) de cada uno de los principales motivos de atención por departamento lo definió un grupo de expertos en el área respectiva (Médico Familiar, Médico Epidemiólogo, Químico, Médico Radiólogo).

- La cantidad de insumos se definió para un tratamiento completo en los procesos agudos y en el caso de procesos crónico degenerativos (diabetes, hipertensión) se estimó para un mes de tratamiento.

- El costo unitario por insumo se determinó a partir de los registros de compras del área administrativa.

- El costo por insumo para cada intervención se determinó multiplicando el costo unitario por la cantidad de insumos definidos.

- El costo por tipo de intervención se estimó a partir de la suma del costo total por insumos.

\section{Costo unitario por departamento y motivo de atención}

Se determinó con la suma del costo unitario fijo y el costo unitario variable.

- La suma del costo unitario fijo por departamento y el costo unitario variable por inter- 
vención permitió conocer el costo unitario por motivo de atención y departamento.

- En cada departamento el costo unitario fijo fue el mismo y el costo unitario variable fue distinto para cada intervención, estableciendo la diferencia entre las intervenciones realizadas en cada departamento.

Costo unitario por departamento, quinquenio y género

Se determinó relacionando el perfil de uso y el costo de la atención.

- Por cada uno de los departamentos finales se identificaron los motivos de atención por quinquenio y género, así como los costos unitarios.

- Se identificó la prevalencia de cada uno de los motivos de atención al interior del quinquenio.

- Se estimó el costo unitario en base al promedio de los costos unitarios por motivo ajustado por la prevalencia.

\section{Costo anual per cápita por género}

Se estableció como el promedio de los costos unitarios ajustados por la prevalencia.

- Se estimó el promedio de los costos unitarios por departamento, quinquenio y género para cada uno de los departamentos finales.

- La suma de los costos de todos los departamentos finales para hombres y mujeres se constituyó como el costo anual per cápita.

\section{Resultados}

En mujeres la consulta de Medicina Familiar es el departamento con promedio de uso más alto, 2.75 en población total y 4.19 en población usuaria. El departamento con promedio de uso más bajo es Trabajo Social, 0.07 en población total y 1.05 en población usuaria. En el Tabla 1 se presenta el promedio de uso de cada uno de los departamentos estudiados.

En hombres la consulta de Medicina Familiar reporta el promedio de uso más alto, 2.27 en población total y 3.89 en población usuaria. El departamento con promedio de uso más bajo es la consulta de Planificación Familiar. En el Tabla 2 se presenta el promedio de uso para el resto de los departamentos.

El departamento con costo unitario más alto es la consulta de Urgencias (US \$47.86) y el más bajo el estudio de Laboratorio (US \$4.96). En el Tabla 3 se presentan los costos unitarios por departamento y género.

En mujeres el costo anual per cápita es de US $\$ 73.24$ en población total y de US \$293.25 en población usuária. En el Tabla 4 se presentan los costos por departamento.

En hombres el costo anual per cápita en población total es US $\$ 53.11$ y en población usuaria US \$258.99. En el Tabla 5 se presenta el costo de cada uno de los departamentos.

Tabla 1. Perfil de uso anual en mujeres por tipo de departamento.

\begin{tabular}{|c|c|c|c|c|c|c|}
\hline \multirow{3}{*}{ Departamento } & \multicolumn{3}{|c|}{ Población total ${ }^{*}$} & \multicolumn{3}{|c|}{ Población usuaria ${ }^{* *}$} \\
\hline & \multirow[b]{2}{*}{ Promedio } & \multicolumn{2}{|c|}{ IC 95\% } & \multirow[b]{2}{*}{ Promedio } & \multicolumn{2}{|c|}{ IC $95 \%$} \\
\hline & & Mínimo & Máximo & & Mínimo & Máximo \\
\hline Consulta Medicina Familiar & 2.75 & 0.60 & 7.26 & 4.19 & 3.92 & 4.46 \\
\hline Enfermera Materno Infantil & 0.11 & 0.01 & 0.25 & 1.77 & 1.55 & 1.99 \\
\hline Consulta Estomatología & 0.22 & 0.01 & 0.63 & 1.58 & 1.37 & 1.78 \\
\hline Trabajo Social & 0.07 & 0.00 & 0.17 & 1.05 & 0.98 & 1.12 \\
\hline Consulta Medicina Preventiva & 0.20 & 0.02 & 0.46 & 1.14 & 1.08 & 1.19 \\
\hline Estudio Laboratorio & 1.24 & 0.10 & 3.32 & 3.91 & 3.58 & 4.25 \\
\hline Estudio Rayos X & 0.11 & 0.00 & 0.37 & 1.36 & 1.20 & 1.53 \\
\hline Consulta Urgencias & 0.12 & 0.00 & 0.31 & 1.17 & 1.07 & 1.28 \\
\hline Consulta Nutrición & 0.08 & 0.00 & 0.30 & 1.19 & 0.97 & 1.40 \\
\hline Consulta Planificación Familiar & 0.11 & 0.00 & 0.43 & 1.55 & 1.34 & 1.75 \\
\hline
\end{tabular}

"Toda la población, independientemente de que haya hecho uso del departamento durante un año; " Población que utilizó al menos una vez al año el departamento. 
Tabla 2. Perfil de uso anual en hombres por tipo de departamento.

\begin{tabular}{|c|c|c|c|c|c|c|}
\hline \multirow{3}{*}{ Departamento } & \multicolumn{3}{|c|}{ Población total ${ }^{*}$} & \multicolumn{3}{|c|}{ Población usuaria ${ }^{* *}$} \\
\hline & \multirow[b]{2}{*}{ Promedio } & \multicolumn{2}{|c|}{ IC $95 \%$} & \multirow[b]{2}{*}{ Promedio } & \multicolumn{2}{|c|}{ IC $95 \%$} \\
\hline & & Mínimo & Máximo & & Mínimo & Máximo \\
\hline Consulta Medicina Familiar & 2.27 & 0.13 & 8.08 & 3.89 & 3.42 & 4.35 \\
\hline Enfermera Materno Infantil & 0.05 & 0.01 & 0.09 & 1.98 & 1.27 & 2.68 \\
\hline Consulta Estomatología & 0.12 & 0.00 & 0.48 & 1.44 & 1.19 & 1.69 \\
\hline Trabajo Social & 0.04 & 0.00 & 0.08 & 1.18 & 0.89 & 1.48 \\
\hline Consulta Medicina Preventiva & 0.12 & 0.02 & 0.58 & 1.12 & 1.03 & 1.22 \\
\hline Estudio Laboratorio & 0.64 & 0.00 & 1.94 & 2.84 & 2.39 & 3.28 \\
\hline Estudio Rayos X & 0.06 & 0.00 & 0.23 & 1.19 & 0.87 & 1.52 \\
\hline Consulta Urgencias & 0.09 & 0.00 & 0.72 & 1.18 & 0.98 & 1.38 \\
\hline Consulta Nutrición & 0.03 & 0.00 & 0.18 & 1.25 & 0.86 & 1.64 \\
\hline Consulta Planificación Familiar & 0.00 & 0.00 & 0.00 & 0.00 & 0.00 & 0.00 \\
\hline
\end{tabular}

*Toda la población, independientemente de que haya hecho uso del departamento durante un año; * Población que utilizó al menos una vez al año el departamento.

\section{Discusión}

En este trabajo el análisis de los costos se presenta por género por considerar que es el abordaje que ofrece la mejor perspectiva de sistema.

El costo se puede calcular para un evento específico, es decir una consulta o un estudio de laboratorio e identificarse como costo unitario, pero esto es de poca utilidad si se desea conocer los recursos necesarios para atender las demandas de salud de un grupo poblacional. Por ello la metodología propuesta cuenta con dos grandes apartados, el costo de la atención y el perfil de uso, el ajuste que se hizo del primero a partir del segundo permitió generar el costo per cápita de la atención y así conocer los recursos que se requieren para generar servicios y realizar actividades de planeación.

Es una realidad que la metodología propuesta no es la única que se puede emplear ${ }^{4,5,15}$, y también es cierto que dependiendo de la metodología elegida los costos presentarán variaciones, pero independientemente del método, el costo de la atención identificado es el real para la institución de salud y se convierte en la herramienta que le permite hacer frente a la competencia en la estructura de mercado que se encuentra inmersa. Desde esta perspectiva el costo calculado en este estudio es el verdadero para la institución de salud que se analiza.

Más allá de que los resultados se presentan por género, al interior de estos existen dos vertientes, en la primera se contempla la población total, incluida aquella que no refirió uso de los departamentos analizados, en tanto la segunda
Tabla 3. Costo unitario por tipo de departamento y género.

\begin{tabular}{lcc}
\hline \multirow{2}{*}{\multicolumn{1}{c}{ Departamento }} & \multicolumn{2}{c}{ Costo unitario } \\
\cline { 2 - 3 } & Hombres & Mujeres \\
\hline Consulta Medicina Familiar & 17.24 & 17.25 \\
Enfermera Materno Infantil & 12.93 & 12.93 \\
Consulta Estomatología & 26.50 & 26.57 \\
Trabajo Social & 17.15 & 17.15 \\
Consulta Medicina Preventiva & 6.91 & 6.87 \\
Estudio Laboratorio & 4.96 & 5.00 \\
Estudio Rayos X & 14.10 & 14.12 \\
Consulta Urgencias & 47.86 & 47.62 \\
Consulta Nutrición & 10.18 & 10.18 \\
Consulta Planificación Familiar & 15.32 & 15.32 \\
\end{tabular}

Los costos se expresan en dólares estadounidenses (10.9608).

contempla exclusivamente a los pacientes que utilizaron al menos en una ocasión el departamento en estudio. El empleo de esta información tiene fines diferentes, la primera está más orientada a la estimación de los recursos para una población determinada en un periodo de tiempo, en tanto la segunda es de utilidad para estimar el costo de la atención de un individuo específico, situación propicia para el cálculo de seguros individuales de gastos médicos.

La metodología propuesta para estimar el costo basada en la departamentalización ajustada por productividad, con asignación ponderal de los gastos de los departamentos generales a los finales, permite tener un costo más cercano a 
la realidad, ya que no desprecia el costo de los tiempos muertos, este costo lo incorpora a los costos de la atención, de no ser así la estimación estaría sesgada y ofrecería un costo inferior al verdadero.

El perfil de uso tanto para hombres como mujeres es mayor cuando se compara la población que hace uso de los servicios de salud al menos en una ocasión, con la población general, esto es totalmente lógico como también resulta lógico que los promedios de uso en población usuaria sean siempre mayores a uno. La impor- tancia de presentar esta información se encuentra en el hecho de que los valores mostrados son los que permiten ajustar el costo unitario. Así se encuentra que al calcular el costo per cápita por departamento, costos unitarios altos se reducen al ajustarlos por el perfil de uso y costos unitarios bajos se incrementan.

Los costos reportados son más altos para la población identificada como usuaria comparada con la población total, esto tiene explicación si se considera que el presupuesto asignado se distribuye en un menor número de población, pero

Tabla 4. Costo anual per cápita en mujeres por tipo de departamento.

\begin{tabular}{|c|c|c|c|c|c|c|}
\hline \multirow{3}{*}{ Departamento } & \multicolumn{3}{|c|}{ Población total } & \multicolumn{3}{|c|}{ Población usuaria ${ }^{* *}$} \\
\hline & \multirow[b]{2}{*}{ Promedio } & \multicolumn{2}{|c|}{ IC 95\% } & \multirow[b]{2}{*}{ Promedio } & \multicolumn{2}{|c|}{ IC $95 \%$} \\
\hline & & Mínimo & Máximo & & Mínimo & Máximo \\
\hline Consulta Medicina Familiar & 47.43 & 10.35 & 125.21 & 72.24 & 67.52 & 76.96 \\
\hline Enfermera Materno Infantil & 1.42 & 0.13 & 3.23 & 22.90 & 20.02 & 25.78 \\
\hline Consulta Estomatología & 5.84 & 0.27 & 16.74 & 41.85 & 36.33 & 47.37 \\
\hline Trabajo Social & 1.20 & 0.00 & 2.92 & 17.96 & 16.77 & 19.15 \\
\hline Consulta Medicina Preventiva & 1.37 & 0.14 & 3.16 & 7.82 & 7.43 & 8.21 \\
\hline Estudios Laboratorio & 6.20 & 0.50 & 16.61 & 19.58 & 17.92 & 21.24 \\
\hline Estudios Rayos X & 1.55 & 0.00 & 5.22 & 19.24 & 16.91 & 21.56 \\
\hline Consulta Urgencias & 5.71 & 0.00 & 14.76 & 55.88 & 50.75 & 61.02 \\
\hline Consulta Nutrición & 0.81 & 0.00 & 3.05 & 12.08 & 9.92 & 14.25 \\
\hline Consulta Planificación Familiar & 1.68 & 0.00 & 6.59 & 23.70 & 20.54 & 26.86 \\
\hline Global & 73.24 & 11.38 & 197.49 & 293.25 & 264.11 & 322.39 \\
\hline
\end{tabular}

* Toda la población, independientemente de que haya hecho uso del departamento durante un año; ${ }^{* *}$ Población que utilizó al menos una vez al año el departamento. Los costos se expresan en dólares estadounidenses (10.9608).

Tabla 5. Costo anual per cápita en hombres por tipo de departamento.

\begin{tabular}{|c|c|c|c|c|c|c|}
\hline \multirow{3}{*}{ Departamento } & \multicolumn{3}{|c|}{ Población total $^{*}$} & \multicolumn{3}{|c|}{ Población usuaria ${ }^{* *}$} \\
\hline & \multirow[b]{2}{*}{ Promedio } & \multicolumn{2}{|c|}{ IC 95\% } & \multirow[b]{2}{*}{ Promedio } & \multicolumn{2}{|c|}{ IC 95\% } \\
\hline & & Mínimo & Máximo & & Mínimo & Máximo \\
\hline Consulta Medicina Familiar & 39.13 & 2.24 & 139.28 & 66.99 & 59.04 & 74.94 \\
\hline Enfermera Materno Infantil & 0.65 & 0.13 & 1.16 & 25.54 & 16.39 & 34.69 \\
\hline Consulta Estomatología & 3.18 & 0.00 & 12.72 & 38.15 & 31.50 & 44.81 \\
\hline Trabajo Social & 0.69 & 0.00 & 1.37 & 20.27 & 15.22 & 25.32 \\
\hline Consulta Medicina Preventiva & 0.83 & 0.14 & 4.01 & 7.76 & 7.11 & 8.40 \\
\hline Estudios Laboratorio & 3.18 & 0.00 & 9.63 & 14.08 & 11.88 & 16.27 \\
\hline Estudios Rayos X & 0.85 & 0.00 & 3.24 & 16.81 & 12.25 & 21.37 \\
\hline Consulta Urgencias & 4.31 & 0.00 & 34.46 & 56.68 & 47.10 & 66.25 \\
\hline Consulta Nutrición & 0.31 & 0.00 & 1.83 & 12.72 & 8.78 & 16.66 \\
\hline Consulta Planificación Familiar & 0.00 & 0.00 & 0.00 & 0.00 & 0.00 & 0.00 \\
\hline Global & 53.11 & 2.51 & 207.71 & 258.99 & 209.27 & 308.71 \\
\hline
\end{tabular}

* Toda la población, independientemente de que haya hecho uso del departamento durante un año; ${ }^{* *}$ Población que utilizó al menos una vez al año el departamento. Los costos se expresan en dólares estadounidenses (10.9608). 
ya se comentó en párrafos anteriores que el uso de esta información tiene diferente aplicación, por lo que debe ser analizada en ese contexto.

Se ha estimado que el gasto en salud per cápita en México corresponde a (US) \$583, cantidad muy por debajo del promedio reportado para los países de la Organización para la Cooperación Económica y el Desarrollo (OCDE) (US $\$ 2,307)^{16}$. Sin embargo existen otras fuentes más conservadoras, las cuales estimaron para el año 2000 que el gasto per cápita para instituciones de seguridad social correspondía a \$1,884 (pesos mexicanos), en tanto para las instituciones con población no asegurada $\$ 793$ (pesos mexicanos $)^{17}$. Valores aun superiores a los presentados en este trabajo para población usuaria, (US) \$73 y (US) \$53 en mujeres y hombres respectivamente, pero es menester señalar que estas cifras corresponden al gasto per cápita para primer nivel de atención, cantidades que representan aproximadamente el 23\% del gasto per cápita total. La importancia de esta información estriba en el aporte al conocimiento de los costos per cápita para el primer nivel de atención.

En conclusión, independientemente del comportamiento de los costos per cápita entre los departamentos, el resultado final revela un mayor costo per cápita en las mujeres que en los hombres, información valiosa en el proceso de planeación de los servicios de salud.

\section{Colaboradores}

E Villarreal-Ríos, MC Esparza, LG Rodríguez, LM González, ERV Daza, GT Labra, AP Veja, MTR Martínez, RA Rojas y MJ Durán participaron conjuntamente en las diferentes etapas de este estúdio y son igualmente responsables del artículo.

\section{Referencias}

1. Meerhoff R. Descentralización, financiamiento, costeo y autogestión de establecimientos de salud: una visión integrada. Washington, D.C.: Organización Panamericana de la Salud; 1997.

2. Drummond M. Métodos para la evaluación económica de los programas de atención de la salud. Madrid: Ediciones Díaz de Santos; 1991.

3. Argentina. Ministerio de Economía, Ministerio de Salud. Estimación del gasto en salud. Argentina. Año 1997 y proyecciones 1997-2000. Buenos Aires; 2001. [consultada 2008 ago 1]. Disponible en: http:// www.mecon.gov.ar/secpro/dir_cn/gsalud.pdf

4. Pellegrino A. Métodos contables de costeo. Facultad de Ciencias Económicas. Universidad de Cuyo. [consultada 2008 ago 1]. Disponible en: http://fce. uncu.edu.ar/investigacion/Jornadas/Jornadas2000/ Jornadas2000/Contables/PellegrinoA/Jornadas.pdf

5. Castellanos EJ. Revisión crítica al ABC y a los "nuevos métodos de costeo". Revista Contaduría y Administración 2003; 209:47-59.

6. Zambrano CM, Zurita GB. Gasto de la atención hospitalaria otorgada a derechohabientes del IMSS mayores de 60 años de edad. In: Martínez SH, Villasís KMA, Torres LJ, Gómez DA, organizadores. Las múltiples facetas de la investigación en Salud 4. México: Instituto Mexicano del Seguro Social; 2000. p. 153-170.

7. Borja AVH, Aguilar MG, Rivera FC, Guzmán CJ, Durcodoy MJEA, Sánchez RFR, Juárez PCA. Costo de la atención médica en enfermedades con alta mortalidad en trabajadores asegurados. In: Martínez SH, Villasís KMA, Torres LJ, Gómez DA, organizadores. Las múltiples facetas de la investigación en Salud 4. México: Instituto Mexicano del Seguro Social; 2000. p. 197-211. 
8. Villarreal-Ríos E, Salinas MAM, Medina JA, Garza-Elizondo ME, Núñez-Rocha GM, Chuy-Díaz E. The cost of diabetes mellitus and its impact on health spending in México. Archives of Medical Research 2000; 31:511-514.

9. Villarreal-Ríos E, Mathew QA, Salinas MAM, Garza EME, Núñez RGM, Gallegos HM. Costo de la atención de la hipertensión arterial y su impacto en el presupuesto destinado a la salud en México. $\mathrm{Sa}$ lud Publica Mex 2002; 1:7-13.

10. Villarreal-Ríos E, Martínez GL, Galicia RL, Vargas DER. El sistema de salud, perfil de uso y costo en primer nivel de atención. México: Universidad $\mathrm{Au}-$ tónoma de Querétaro; 2007.

11. World Health Organization. The World Health report 2005: make every mother and child count. Paris: World Health Organization; 2005.

12. Arzoz J, Knaul F. Inequidad en el gasto del gobierno en salud. In: Caleidoscopio de la salud. 185194. [consultada 2007 julio 26]. Disponible en: http:/ /www.funsalud.org.mx/casesalud/caleidoscopio/ $13 \% 20$ Inequidad $\% 20$ en $\% 20$ el $\% 20$ gasto.pdf

13. Zurita B, Zambrano M, Ramírez T, Villarreal-Ríos E, Escobedo de la Peña J, Rodríguez G, Orozco R, Acevedo F, Carlos C, Shettino A, García A. Gasto en atención médica 2001. In: Muñoz O, Durán L, Garduño J, Soto H, organizadores. Economía de la salud: seminario internacional. México: Instituto Mexicano del Seguro Social; 2003. p.115-124.

14. Instituto Nacional de Geografía Estadística e Informática. Mujeres y Hombres en México. Quinta Edición. Indicadores Sociodemográficos de México 1930-2000. [consultada 2007 julio 26]. Disponible en: http://www.inegi.org.mx/prod_serv/contenidos/ español/bvinegi/productos/integracion/socio demograficos/indisociodem/2001/indi2001.pdf

15. Tobar F, Ventura G, Montiel L, Falbo R. El gasto en salud en Argentina y su método. Buenos Aires; 2000. [consultada 2007 julio 4]. Disponible en: http:// www.who.int/nha/docs/es/Argentina_NHAreport_ spanish.pdf

16. Eco-Salud OCDE 2005. Como se encuentra México comparativamente. [consultada 2007 julio 4]. Disponible en: http://www.oecd.org/dataoecd/51/15/ 35140519.pdf

17. Coordinación General de Planeación Estratégica. Gasto público en salud 1999-2000. Síntesis ejecutiva 3. Secretaría de Salud. Coordinación General de Planeación Estratégica. Dirección General de Información y Evaluación del Desempeño. [consultada 2007 julio 4]. Disponible en: http://evaluación.sañud.gob. $\mathrm{mx} /$ publicaciones/gastopublico.pdf

Artigo apresentado em 14/08/2007

Aprovado em 30/06/2008

Versão final apresentada em 30/10/2008 